\title{
25 Research Soure \\ Effect of pH on the Conformational Transition of Silk Fibroin in Aqueous Solution Monitored by Thioflavin-T Fluorescence
}

Ben Jia

Taiyuan University of Technology School of Materials Science and Engineering

Lan Jia ( $\nabla$ jialan@tyut.edu.cn )

Taiyuan University of Technology https://orcid.org/0000-0001-7005-5873

Jingxin Zhu

Taiyuan University of Technology

\section{Research Article}

Keywords: Conformational transition, Fluorescence spectroscopy, Protein structures, Silk fibroin,

Thioflavin-T

Posted Date: July 16th, 2021

DOI: https://doi.org/10.21203/rs.3.rs-699204/v1

License: (9) This work is licensed under a Creative Commons Attribution 4.0 International License.

Read Full License

Version of Record: A version of this preprint was published at Journal of Fluorescence on December 2nd, 2021. See the published version at https://doi.org/10.1007/s10895-021-02841-x. 


\section{Abstract}

In this work, the potential application of the fluorescence dye Thioflavin-T (ThT), which can specifically bind to amyloid, as a powerful tool for monitoring secondary structure transitions of silk fibroin (SF) induced by $\mathrm{pH}$ was examined. Results showed that ThT emission intensities substantially increased when $\mathrm{pH}$ decreased from 6.8 to 4.8. This increase may be due to conformational transitions from random coil to $\beta$-sheet. The morphology and secondary structure of SF were also investigated via TEM, AFM and circular dichroism spectroscopy. The information obtained herein can be utilized not only for the development of convenient and efficient noninvasive method for monitoring the assembly behavior of SF in aqueous solution but also for in vitro fluorescence imaging.

\section{Introduction}

The self-assembly of natural proteins or designed peptides into fibrillar structures based on $\beta$-sheet conformation is a ubiquitous and important phenomenon[1-3]. These structures typically include conserved peptide fragments from silk proteins and critical peptide fragments from amyloid proteins related to degenerative diseases. Owing to the adjustable mechanical properties and biodegradability of silk protein-based materials that are related closely to the content of $\beta$-sheet crystallinity, they are promising candidates in material sciences and biotechnology $[4,5]$.

Various methods, such as Fourier transform infrared spectroscopy (FTIR)[6, 7], Raman spectroscopy[8], circular dichroism (CD) [9], nuclear magnetic resonance (NMR) spectroscopy[9, 10], X-ray scattering[11], rheology[12,13], and fluorescence spectroscopy[14], have been used to study the transition from random coil and/or helix to $\beta$-sheet in fibroin solutions. However, most of the studies that adopted these methods yielded static rather than dynamic data as they only described the conformations of silk protein before and after natural spinning or under specified conditions. A more useful endeavor is to follow protein conformational changes continuously over time while they are induced by various factors, such as shear force, $\mathrm{pH}$, metallic ions, temperature change, and low dielectric constant solvents. This undertaking would yield important data on the kinetics of conformation transitions that cannot be obtained by static experiments[15]. Thus, a detailed understanding of the sequence of events that occurs as random coil and/or helix conformation changes to $\beta$-sheet in silk proteins can be achieved.

Fluorescence spectroscopy is widely used to interpret protein structure in real time. For example, the intrinsic fluorescence relative changes in tryptophan (Trp) and tyrosine (Tyr) in silk fibroin (SF) can monitor conformational states among silk solution, gel, and scaffold samples[16]. Moreover, 8-anilino-1naphthalene-sulfonic acid (ANS) is utilized as an external probe to investigate the effects of environmental factors in aqueous solutions[17].

Since its first description in 1959[18], Thioflavin-T (ThT) has become the "gold standard" for selectively staining and identifying amyloid fibrils because its fluorescence emission intensifies upon binding to fibrils[19]. Given the similarities in self-assembly into fibrillar structures between SF and amyloid protein, 
the fluorescent dye ThT, which specifically binds to amyloid protein, was utilized herein as an external probe to monitor conformational transitions of SF under different pH conditions. However, ThT is not widely used to monitor the conformational transitions of SF.

Scheme 1

The design rationale is illustrated in Scheme 1. The enhancement of ThT fluorescence emission is associated with elevated $\beta$-sheet contents as $\mathrm{pH}$ decreases from 6.8 to 4.8. This enhancement makes ThT a particularly convenient and suitable tool for monitoring conformational transitions. Intrinsic Trp residues and another commonly used hydrophobic dye, namely, ANS, were also investigated for comparison. The morphologies were observed via transmission electron microscopy (TEM) and atomic force microscopy (AFM). FTIR and CD spectroscopy were performed to monitor the secondary structures of SF.

\section{Materials And Methods \\ 2.1 Materials}

ThT and ANS were purchased from Sigma Co. (USA). Tris (hydroxymethyl) aminomethane (Tris) and 2(N-morpholino) ethanesulfonic acid (MES) were obtained from Shanghai Sangon (Shanghai, China). All other reagents were of analytical grade and used as received. All aqueous solutions were prepared with Milli-Q purification system.

\subsection{Preparation of SF solutions}

Aqueous SF solutions were prepared following the method described in a previous report[13]. In brief, degummed fibers were dissolved in 9.3 M LiBr solutions, and SF solution was dialyzed against distilled water using Slide-a-Lyzer dialysis cassettes (MWCO 3500, Pierce) for $3 \mathrm{~d}$ (triple-distilled water for the last day) until the conductivity of the dialyzed water was close to that of distilled water.

\subsection{Optimization of the probe}

ThT stock solution at $1 \mathrm{mM}$ concentration was prepared by Milli-Q water and filtered through a $0.22 \mu \mathrm{m}$ filter. Afterward, $2 \mathrm{~mL}$ of SF solutions $\left(10 \mathrm{mg} \mathrm{mL}^{-1}\right)$ were mixed with different amounts of ThT stock solution (the final ThT concentration was $2.5,5,7.5,10,20$, and $40 \mu \mathrm{M}$ ). The mixtures were diluted to 4 $\mathrm{mL}$ with water and kept at room temperature in the dark for $2 \mathrm{~h}$.

\subsection{The effect of $\mathrm{pH}$ on the structure change of the SF}

To investigate the conformation change of the SF solutions with respect to $\mathrm{pH}$, the mixtures of $\mathrm{SF}$ and ThT were added to $20 \mathrm{mM}$ Tris-MES buffer with different $\mathrm{pH}(4.8,5.0,5.2,5.6$ and 6.8). The final concentrations of SF and ThT were $5 \mathrm{mg} \mathrm{mL}^{-1}$ and $20 \mu \mathrm{M}$, respectively.

\subsection{Characterizations}


Fluorescence measurements were performed using a Fluoromax-4 fluorescence spectrophotometer (HORIBA, USA). For intrinsic fluorescence spectra, the excitation wavelength was set as $280 \mathrm{~nm}$ and emission spectra were recorded ranged form 300 to 500. For ThT and ANS fluorescence measurements, the samples were excited at $420 \mathrm{~nm}$ and $390 \mathrm{~nm}$, the emission spectra were recorded in the range of 430-650 nm and 400-650 nm respectively. Protein concentration for all the fluorescence experiments was $5 \mathrm{mg} \mathrm{mL}^{-1}$, using slit width of $5 \mathrm{~nm}$ for both excitation and emission.

TEM was performed on a JEM-1200EX (JEOL, Japan). Samples for TEM measurements were prepared by placing a drop of colloidal dispersion on a carbon-coated copper grid, followed by evaporation of the solvent. To prepare the samples for AFM imaging, $2 \mu \mathrm{L}$ of the diluted SF solution was cast on silicon wafer and was allowed to dry in ambient air for $20 \mathrm{~min}$. The morphology of silk fibroin was observed by NX10 AFM (Park Systems, Korea) in air. AFM scan rate was $1.0 \mathrm{~Hz}$ and imaging resolution was $256 \times 256$ pixel.

Samples were freeze-dried completely before being grinded into powder and pressed into pellets with $\mathrm{KBr}$ for measuring infrared spectra with a Fourier transform infrared spectrophotometer (FTIR) (Bruker VERTEX80 V, Germany).

Circular dichroism (CD) spectra were recorded on a MOS-500 spectropolarimeter (Bio-Logic, France) by using a quartz cell with a $1 \mathrm{~cm}$ path length under a nitrogen atmosphere. The spectra of sample solutions ( $5 \mathrm{mg} \mathrm{mL}^{-1}$ ) were obtained from 190 to $260 \mathrm{~nm}$ with a $0.2 \mathrm{~nm}$ step and $2 \mathrm{~s}$ collection times per step, taking three averages.

The fibroin aqueous solutions under different $\mathrm{pHs}$ were kept at ambient temperature for 5 days to ensure the conformational transition for TEM, AFM, FTIR and CD measurements.

\section{Results And Discussion}

\subsection{Optimization of the probe}

Fluorescence spectroscopy is widely used to interpret protein structure at atomic resolution. Aromatic amino acids, e.g., Trp, Tyr, and phenylalanine, offer intrinsic fluorescent probes for monitoring protein conformations, dynamics, and intermolecular interactions. Among them, Trp is the most popular probe for investigating protein structural changes because it is sensitive to the environment[20]. The fluorescence spectroscopy of Trp in SF was evaluated under different $\mathrm{pH}$ conditions to indicate changes in SF conformation. However, as shown in Fig. S1, the emission spectrum barely changed with $\mathrm{pH}$. This result may be attributed to the fact that Trp is more sensitive to the water content of SF because most Trp residues are distributed on the surface of proteins, a property that induces poor sensitivity in aqueous solution. Thus, the external probe might be more suitable for our system.

ThT is perhaps the most widely used amyloid dye as this reagent specifically binds to the $\beta$-sheet structure of fibrils[21,22]. In this study, ThT was used as an external probe to monitor the conformational 
state of SF. As shown in Fig. 1a, the fluorescence intensity of ThT at $485 \mathrm{~nm}$ substantially increased upon binding to the hydrophobic region of SF compared with free ThT. Thus, this phenomenon was qualified for fluorescence assay. The relationship between ThT concentration and fluorescence intensities at 485 $\mathrm{nm}$ of the SF solution is depicted in Fig. 1b. The highest intensity was obtained with $20 \mu \mathrm{M} \mathrm{ThT}$, and it decreased when the concentration exceeded $30 \mu \mathrm{M}$ probably because of the self-quenching effect of excessive ThT. Hence, $20 \mu \mathrm{M}$ ThT was chosen as the optimum concentration and used for further analysis.

\subsection{Effect of $\mathrm{pH}$ on structural changes in SF}

$\mathrm{pH}$ is clearly an important factor in the formation of supramolecular assemblies of Bombyx mori SF because it has an influence on electrostatic interactions[12]. The fluorescence emission spectra of SF at $\mathrm{pH} 4.8,5.0,5.2,5.6$, and 6.8 are given in Fig. 2. These $\mathrm{pH}$ levels represent the conditions at different parts of the silk gland of Bombyx mori: $\mathrm{pH} 4.8$ for the anterior division and $\mathrm{pH} 6.8$ for the posterior division[23].

Emission intensity increased by about 3.6-fold when $\mathrm{pH}$ decreased from 6.8 to 4.8 , indicating the $\beta$-sheet conformation of SF in aqueous solution. Photographs of SF solutions with the ThT probe at pH 4.8, 5.2, and 6.8 displayed the same trend. The fluorescence strategy could be used as a real-time method for monitoring the assembly process. The emission intensities at $485 \mathrm{~nm}$ initially increased with incubation time at $\mathrm{pH} 4.8$ and 5.0 within 5 days and then decreased over time. This result might be ascribed to the aggregated formation and the self-quenching of the ThT dye. By contrast, the intensity scarcely increased at $\mathrm{pH}$ 6.8. The $\mathrm{pH}$ and time dependence of ThT fluorescence was also determined. Results showed that the effect of $\mathrm{pH}$ and incubation time were negligible for pure ThT (Fig. S2).

ANS is also a frequently used extrinsic fluorescence probe for exploring changes in hydrophobic regions during conformational transitions of SF. Its fluorescence behavior upon binding to SF was also investigated for comparison. The fluorescence spectrum of pure ANS exhibited a wide band with very low fluorescence intensity and an emission maximum of $542 \mathrm{~nm}$ in polar environments (Fig. S3a). The fluorescence intensity of ANS evidently increased in the presence of SF, which was accompanied by shifting of the emission maximum to $471 \mathrm{~nm}$. The emission intensity of ANS at $470 \mathrm{~nm}$ also increased when $\mathrm{pH}$ decreased from 6.8 to 4.8 . However, compared with ANS, the ThT probe showed better sensitivity as the fluorescence enhancement was more obvious. Compared with free ThT, the fluorescence of the SF solution with the ThT probe increased by about 25 -fold at $\mathrm{pH} 4.8$, whereas the fluorescence of the SF solution with the ANS probe increased by about 7-fold only (Fig. S3b). The difference in this phenomenon might be ascribed to the possibility that ANS was sensitive to the hydrophobic environment, whereas ThT was bound to the $\beta$-sheet structure. Moreover, SF (PI 4.5) is negatively charged at $\mathrm{pH} 4.8$ to 6.8 , whereas ANS has a negative sulfonate ion in aqueous solution[24]. The repulsion force between SF and ANS also made them difficult to be closely bound.

The assembly process was further investigated via TEM. As $\mathrm{pH}$ was decreased, the morphology of SF transitioned from spherical to dendritic aggregates. At pH 4.8, the aggregates formed by rod-like structures appeared (Fig. 3a and Fig. 3b), whereas at pH 6.8, heterogeneously sized micelles with 
diameters ranging from several tens of nanometers to hundreds of nanometers were observed (Fig. 3d). Previous studies identified micelles at neutral $\mathrm{pH}[23]$. These structures might be assembled extrinsically by the aggregation of solubilized globular protein chains driven by their amino acid hydrophobicity. At pH 5.2, irregular and patchy aggregates can be seen in the image (Fig. 3c).

The results of AFM were basically consistent with those of TEM. At pH 4.8, SF proteins assembled into granules and displayed a tendency to join into bundles of fibrils (Fig. 4a). Moreover, the length of the fibrils reached micron scale. At pH 5.2 and 4.8, only particle-like aggregates can be seen, and the proportion of larger aggregates was higher at pH 5.2.

Differences among the FTIR spectra of SF under different $\mathrm{pH}$ values were not remarkable. As shown in Fig. S4, the peak at 1650 (amide I) and $1545 \mathrm{~cm}^{-1}$ (amide II) were associated with the random coil of SF, whereas the peak around 1630 (amide I), 1530 (amide II), and $1245 \mathrm{~cm}^{-1}$ (amide III) were characteristic of $\beta$-sheet structure. Both random coil and $\beta$-sheet structure existed in the solutions under different $\mathrm{pH}$ values. The peak at $1245 \mathrm{~cm}^{-1}$ was more obvious at $\mathrm{pH} 4.8$ than at $\mathrm{pH} 6.8$. This result may be attributed to the low $\beta$-sheet content in the aqueous solution.

$\mathrm{CD}$ spectra were used to verify the second structure of $\mathrm{SF}$ under different $\mathrm{pH}$ values. $\mathrm{At} \mathrm{pH}$ 6.8, the $\mathrm{CD}$ spectra demonstrated a strong negative band centered around $193 \mathrm{~nm}$ and a negative one at $215 \mathrm{~nm}$, indicating the dominance of random-coil structure. However, when $\mathrm{pH}$ decreased to 4.8 , the two bands drastically inverted to a positive band at $195 \mathrm{~nm}$ and to a negative band at $215 \mathrm{~nm}$, indicating the emergence of $\beta$-sheet conformation. The $C D$ spectra indicated a decrease in the content of random coil and an increase in $\beta$-sheet.

\section{Conclusion}

In summary, the conformational transitions of $\mathrm{SF}$ in aqueous solution under different $\mathrm{pH}$ values were investigated using ThT, a fluorescence dye that specifically binds to b-sheet. A substantial fluorescence enhancement was demonstrated as $\mathrm{pH}$ decreased. The time dependence of ThT fluorescence was also investigated under different $\mathrm{pH}$ values. The level of fluorescence attributed to $b$-sheet content substantially increased. ThT was more efficient than ANS and intrinsic Trp residues because it showed a more substantial fluorescence enhancement ratio. This result may be attributed to the ability of ThT to specifically bind to b-sheet. The morphological transition from spherical micelles to rod-like aggregates and fibrils due to a reduction in $\mathrm{pH}$ was observed via TEM and AFM, respectively. The transition of the secondary structure from random coil to $\beta$-sheet during this change was confirmed via CD spectroscopy. Thus, the fluorescence external probe may provide a direct and suitable noninvasive tool for monitoring the assembly behavior of proteins with conformational transitions in aqueous solutions. Owing to its ability to specifically bind to b-sheet, ThT has the potential for bioimaging of silk-based materials in vitro.

\section{Declarations}




\section{Compliance with Ethical Standards}

Acknowledgments This work was supported by the Natural Science Foundation of Shanxi (Grant No. 201801D121360 and 201801D121102).

Conflict of interest The authors declare that they have no competing interests.

Ethics approval No applicable.

Consent to participate No applicable.

Consent for publication No applicable.

Availability of data and material No applicable.

Code availability No applicable.

Authors' contributions: "Conceptualization, resources, writing-review and editing, project administration and funding acquisition, L. Jia; methodology and supervision, J. X. Zhu; investigation, data curation and writing-original draft preparation, $\mathrm{B}$. Jia. All authors have read and agreed to the published version of the manuscript."

\section{References}

1. Dobson CM (2003) Protein folding and misfolding. Nature 426:884-890

2. Stefani M, Dobson CM (2003) Protein aggregation and aggregate toxicity: new insights into protein folding, misfolding diseases and biological evolution. Journal of molecular medicine (Berlin, Germany) 81 :678-699. doi:10.1007/s00109-003-0464-5

3. Lu Q, Zhu H-S, Zhang C-C, Zhang F, Zhang B, Kaplan DL (2012) Silk Self-Assembly Mechanisms and Control From Thermodynamics to Kinetics. Biomacromolecules 13: 826832.doi:10.1021/bm201731e

4. Bai S-M, Zhang X-L, Lu Q, Sheng W-Q, Liu L-J, Dong B-J, Kaplan DL, Zhu H-S (2014) Reversible Hydrogel-Solution System of Silk with High Beta-Sheet Content. Biomacromolecules 15: 3044-3051. doi:10.1021/bm500662z

5. Pei Y-Z, Liu X, Liu S-S, Lu Q, Liu J, Kaplan DL, Zhu H-S (2015) A mild process to design silk scaffolds with reduced $\beta$-sheet structure and various topographies at the nanometer scale. Biophysical Acta Biomaterialia 13: 168-176. doi:10.1016/j.actbio.2014.11.016

6. Chen X, Shao Z-Z, Marinkovic NS, Miller LM, Zhou P, Chance MR (2001) Conformation transition kinetics of regenerated Bombyx mori silk fibroin membrane monitored by time-resolved FTIR spectroscopy. Biophysical chemistry 89: 25-34.doi:10.1016/S0301-4622(00)00213-1

7. Ling S-J, Qi Z-M, Knight DP, Shao Z-Z, Chen X (2011) Synchrotron FTIR Microspectroscopy of Single Natural Silk Fibers. Biomacromolecules 12: 3344-3349.doi:10.1021/bm2006032 
8. Zhou P, Xun X, Knight DP, Zong X-H, Deng F, Yao W-H (2004) Effects of pH and Calcium lons on the Conformational Transitions in Silk Fibroin Using 2D Raman Correlation Spectroscopy and ${ }^{13} \mathrm{C}$ SolidState NMR \pm . Biochemistry 43: 11302-11311.doi:10.1021/bi049344i

9. Ha SW, Asakura T, Kishore R (2006) Distinctive Influence of Two Hexafluoro Solvents on the Structural Stabilization of Bombyx mori Silk Fibroin Protein and Its Derived Peptides: ${ }^{13} \mathrm{C}$ NMR and CD Studies. Biomacromolecules 7: 18-23.doi:10.1021/bm050783m

10. Asakura T, Okushita K, Williamson MP (2015) Analysis of the Structure of Bombyx mori Silk Fibroin by NMR. Macromolecules 48: 2345-2357.doi:10.1021/acs.macromol.5b00160

11. Rossle M, Panine P, Urban VS, Riekel C (2004) Structural evolution of regenerated silk fibroin under shear: Combined wide-and small-angle x-ray scattering experiments using synchrotron radiation. Biopolymers 74: 316-327.doi:10.1002/bip.20083

12. Terry AE, Knight DP, Porter D, Vollrath F (2004) pH Induced Changes in the Rheology of Silk Fibroin Solution from the Middle Division of Bombyx mori Silkworm. Biomacromolecules 5: 768772.doi:10.1021/bm034381v

13. Zhu J-X, Zhang Y-P, Shao H-L, Hu X-C (2008) Electrospinning and rheology of regenerated Bombyx mori silk fibroin aqueous solutions: The effects of $\mathrm{pH}$ and concentration. Polymer 49: 28802885.doi:10.1016/j.polymer.2008.04.049

14. Royer CA (2006) Probing Protein Folding and Conformational Transitions with Fluorescence. Chemical Reviews 106: 1769-1784.doi:10.1021/cr0404390

15. Chen X, Shao Z-Z, Knight DP, Vollrath F (2007) Conformation transition kinetics of Bombyx mori silk protein. Proteins-Structure Function and Bioinformatics 68: 223-231.doi:10.1002/prot.21414

16. Tsai I, Greiner C, Wong C, Defelice J, Kaplan DL (2007) Intrinsic fluorescence changes associated with the conformational state of silk fibroin in biomaterial matrices, Optics Express 15: 10431053.doi:10.1364/OE.15.001043

17. Yang Y-H, Shao Z-Z, Chen X, Zhou P (2004) Optical Spectroscopy To Investigate the Structure of Regenerated Bombyx mori Silk Fibroin in Solution. Biomacromolecules 5: 773779.doi:10.1021/bm0343848

18. Vassar PS, Culling CF (1959) Fluorescent stains, with special reference to amyloid and connective tissues. Achives of pathology 68: 487-498.

19. LeVine H, 3rd (1999) Quantification of $\beta$-sheet amyloid fibril structures with thioflavin T. Methods in enzymology 309: 274-284.doi:10.1016/S0076-6879(99)09020-5

20. Vivian JT, Callis PR (2001) Mechanisms of Tryptophan Fluorescence Shifts in Proteins. Biophysical journal 80: 2093-2109.doi:10.1016/S0006-3495(01)76183-8

21. Namioka S, Yoshida N, Konno H, Makabe K (2020) Residue-Specific Binding Mechanisms of Thioflavin T to a Surface of Flat $\beta$-Sheets within a Peptide Self-Assembly Mimic. Biochemistry 59: 2782-2787.doi:10.1021/acs.biochem.0c00280 
22. Groenning M (2010) Binding mode of Thioflavin T and other molecular probes in the context of amyloid fibrils-current status. Journal of chemical biology 3: 1-18.doi:10.1007/s12154-009-0027-5

23. Chen P, Kim HS, Park CY, Kim HS, Chin IJ, Jin HJ (2008) pH-Triggered transition of silk fibroin from spherical micelles to nanofibrils in water. Macromolecular Research 16: 539-

543.doi:10.1007/BF03218556

24. Qu P, Lu H, Yan S-C, Zhou D-Q, Lu Z-H (2009) Investigations of effects of environmental factors in unfolding/refolding pathway of proteins on 8-anilino-1-naphthalene-sulfonic acid (ANS) fluorescence. Journal of Molecular Structure 936: 187-193.doi:10.1016/j.molstruc.2009.07.037

Figures
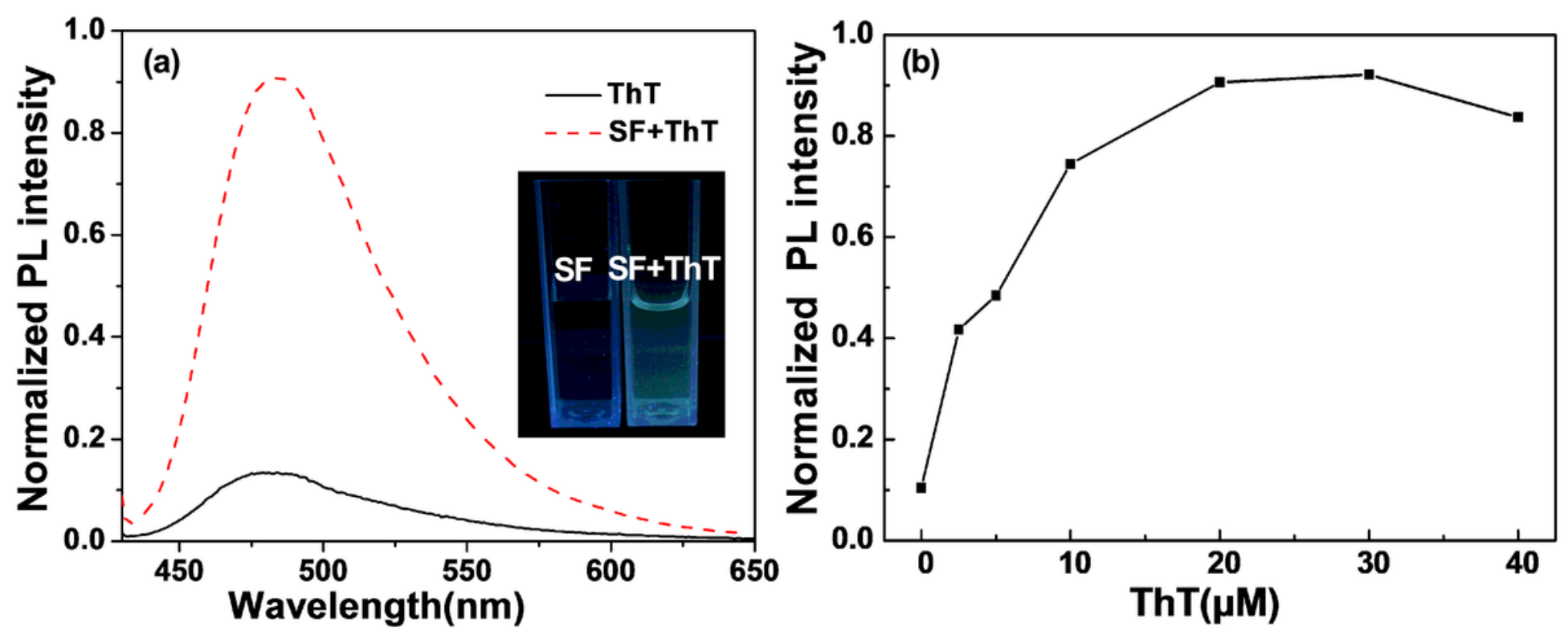

Figure 1

(a) Fluorescence emission spectra of ThT $(20 \mu \mathrm{M})$ and SF+ThT solutions in water. (b) Fluorescence intensity as a function of ThT concentration at $485 \mathrm{~nm}$. 

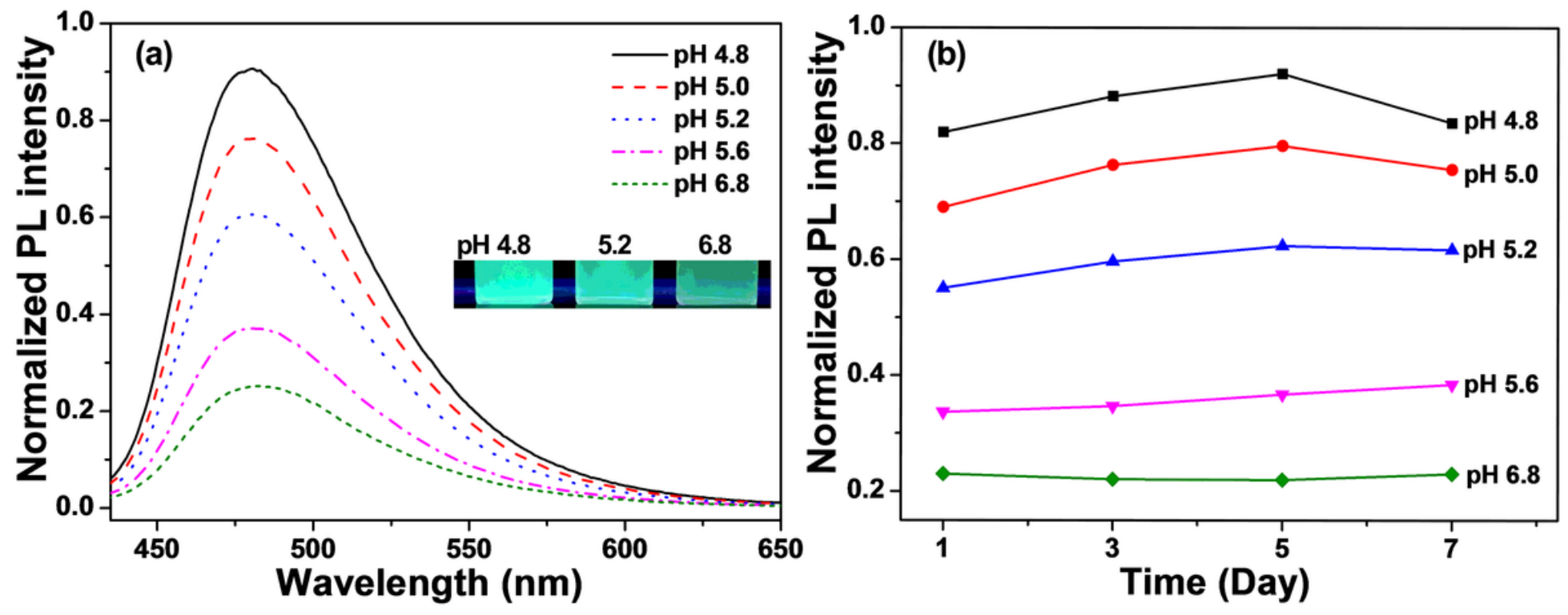

Figure 2

Fluorescence emission spectra of silk fibroin at different $\mathrm{pH}$ and emission intensity at $485 \mathrm{~nm}$ as a function of time.

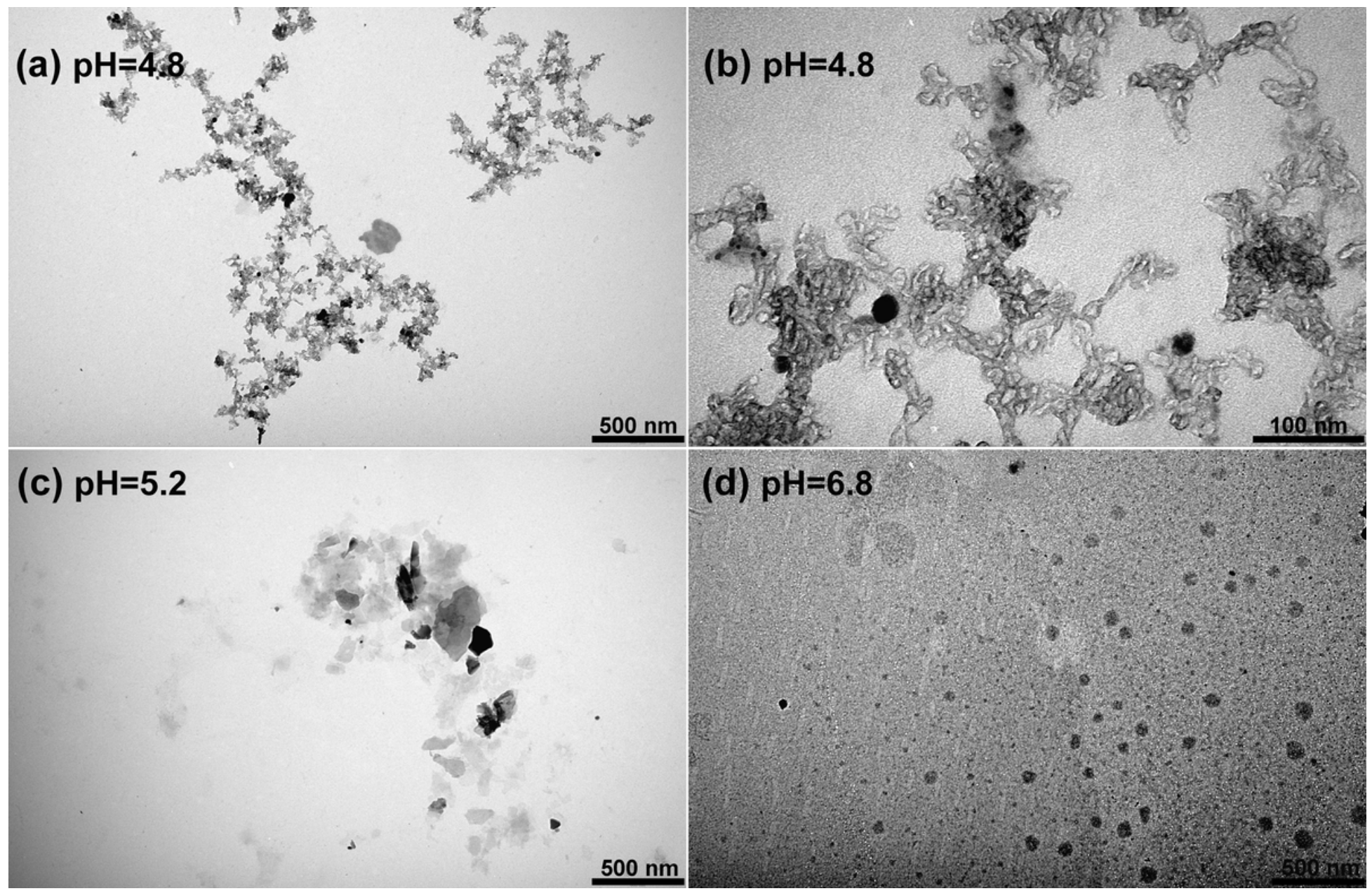

Figure 3 
TEM images SF (5mg mL-1) at different $\mathrm{pH}$ values.

(a) $\mathrm{pH} 4.8$
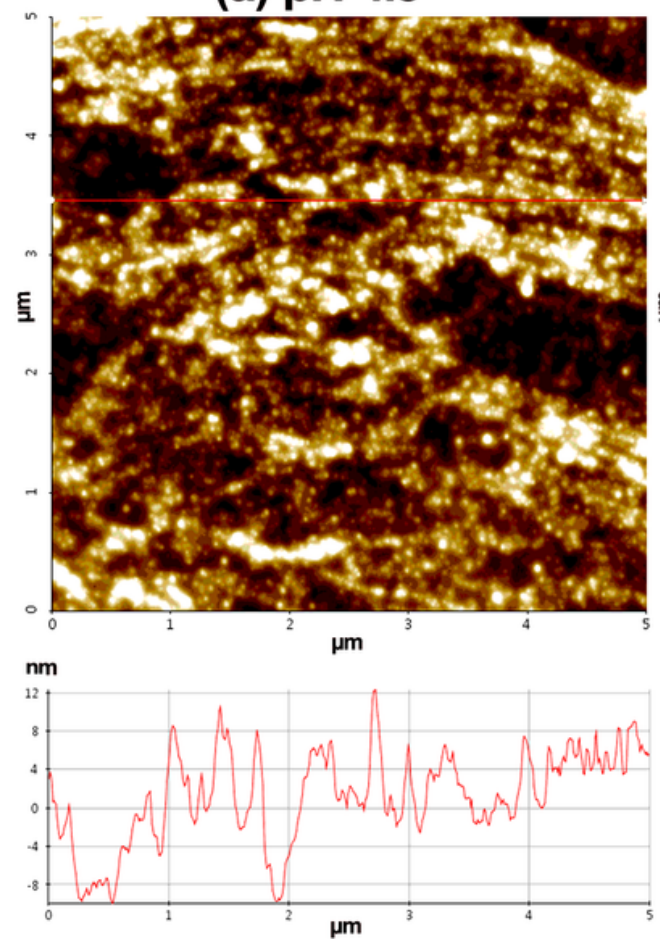

(b) $\mathrm{pH} 5.2$
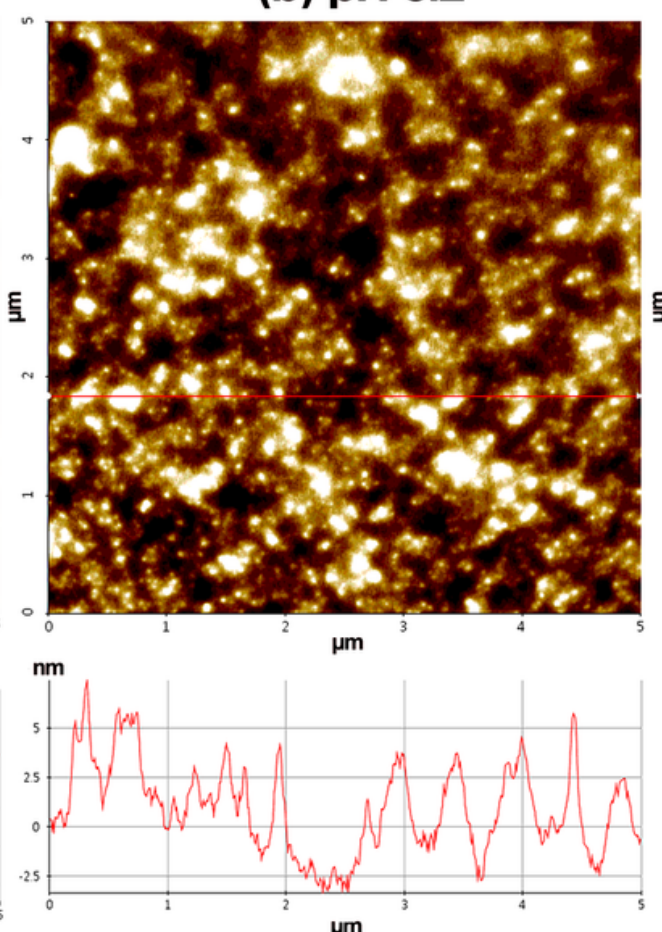

(c) $\mathrm{pH} 6.8$
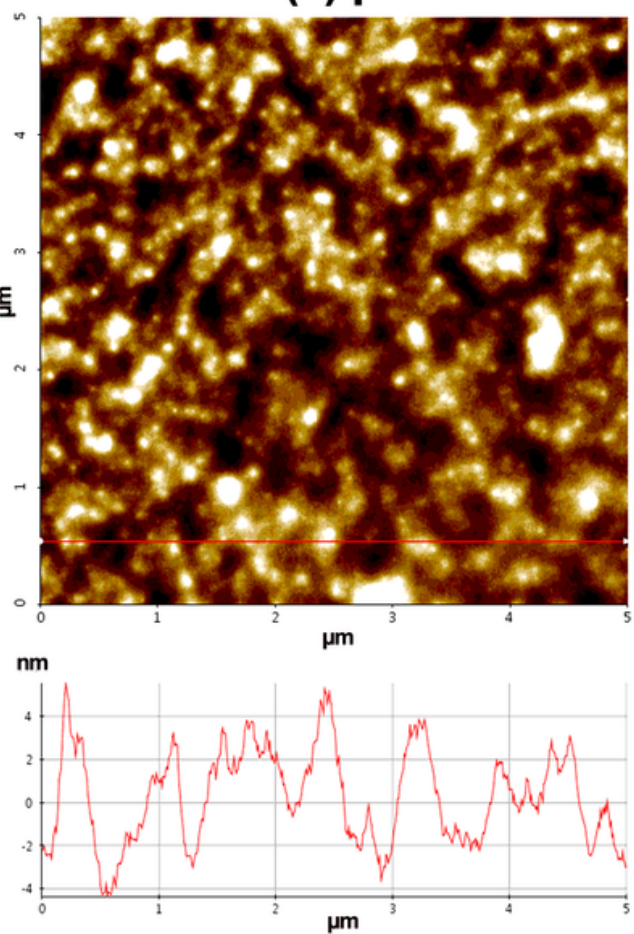

Figure 4

AFM images and results of height analysis of SF at different $\mathrm{pH}$ values. 


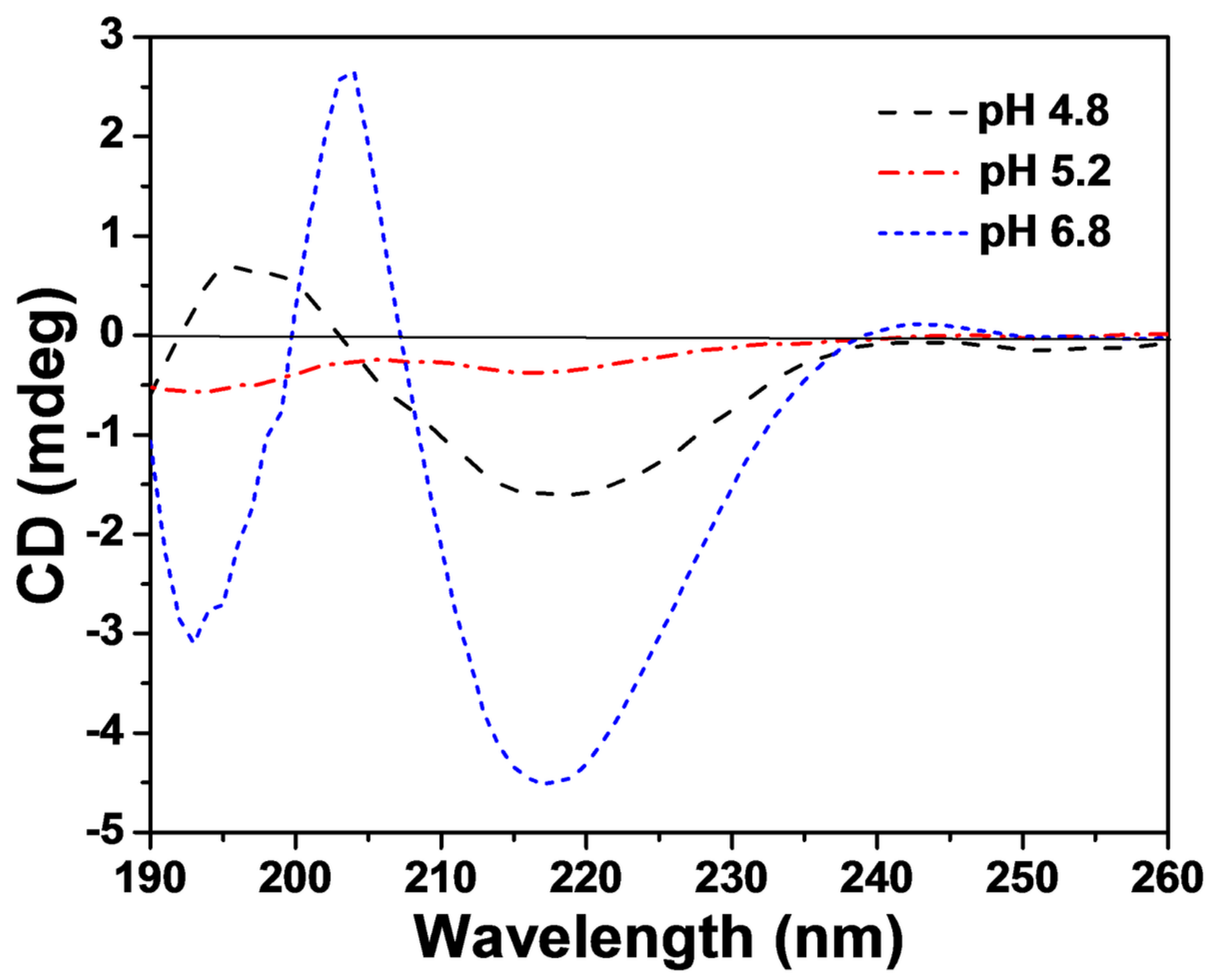

Figure 5

CD spectra of $\mathrm{SF}(5 \mathrm{mg} \mathrm{mL}-1)$ at different $\mathrm{pH}$ values.

\section{Supplementary Files}

This is a list of supplementary files associated with this preprint. Click to download.

- Scheme1.tif

- SupplementaryInformation.docx 